\title{
Dual-wavelength quantum cascade laser for trace gas spectroscopy
}

\author{
J. Jágerská, ${ }^{1}$ P. Jouy, ${ }^{2}$ A. Hugi, ${ }^{2}$ B. Tuzson, ${ }^{1}$ H. Looser, ${ }^{3}$ M. Mangold, ${ }^{1}$ M. Beck, ${ }^{2}$ \\ L. Emmenegger, ${ }^{1}$ and J. Faist ${ }^{2}$ \\ ${ }^{1}$ Laboratory for Air Pollution and Environmental Technology, Empa, Überlandstrasse 129, 8600 Dübendorf, \\ Switzerland \\ ${ }^{2}$ Institute for Quantum Electronics, ETH Zürich, Wolfgang-Pauli-Str. 16, 8093 Zürich, Switzerland \\ ${ }^{3}$ Institute for Aerosol and Sensor Technology, FHNW, Klosterzelgstrasse 2, 5210 Windisch, Switzerland
}

(Received 9 September 2014; accepted 10 October 2014; published online 23 October 2014)

\begin{abstract}
We demonstrate a sequentially operating dual-wavelength quantum cascade laser with electrically separated laser sections, emitting single-mode at 5.25 and $6.25 \mu \mathrm{m}$. Based on a single waveguide ridge, this laser represents a considerable asset to optical sensing and trace gas spectroscopy, as it allows probing multiple gas species with spectrally distant absorption features using conventional optical setups without any beam combining optics. The laser capability was demonstrated in simultaneous $\mathrm{NO}$ and $\mathrm{NO}_{2}$ detection, reaching sub-ppb detection limits and selectivity comparable to conventional high-end spectroscopic systems. (C) 2014 AIP Publishing LLC.

[http://dx.doi.org/10.1063/1.4899061]
\end{abstract}

A unique feature of quantum cascade $(\mathrm{QC})$ lasers $^{1-3}$ is their atomic-like joint density of states originating from the intersubband nature of the lasing transition. In consequence, the active region of a QC laser is transparent on both sides of the resonance, which allows to combine in one waveguide several active regions designed for different emission wavelengths. Such devices, known as heterogeneous QC lasers, have been demonstrated more than a decade ago; ${ }^{4,5}$ however, only due to recently developed predictive physical models ${ }^{6}$ and improvements in fabrication technology designs avoiding spurious cross-absorption have been realized. The stateof-the-art heterogeneous cascade lasers constitute the basis of broadband active regions for external cavity lasers ${ }^{7}$ as well as for mid-infrared (MIR) optical combs. ${ }^{8}$ They can also provide for emission at a number of fixed, spectrally well-separated wavelengths, thus realizing so-called multiwavelength QC lasers. ${ }^{9}$

Several different strategies for achieving single-mode emission with multi-wavelength lasers have been reported. Besides edge emitting ${ }^{10}$ and surface emitting distributedfeedback (DFB) multi-wavelength laser arrays, ${ }^{11}$ aperiodic DFB grating designs, ${ }^{12,13}$ as well as an approach combining two DFB sections on the same waveguide ridge has been reported. ${ }^{14}$ The latter are extremely appealing for applications in laser spectroscopy, since emission of multiple wavelengths within a single optical beam allows for detection of several molecules at a time without the need for couplers or beam combining optics. However, the multi-wavelength lasers of this type were operating up to now only at cryogenic temperatures or simultaneously at all emission wavelengths. Strictly simultaneous operation constitutes a principal drawback for practical applications, since, combined with conventional broadband MIR detectors, it leads to an overlap of the measured spectra and to a cross-talk between the detected gas species.

In this paper, we demonstrate a dual-wavelength $\mathrm{QC}$ laser with electrically separated DFB sections, which introduces the option of addressing each wavelength independently. Moreover, owing to advanced numerical modelling methods applied in the active region design, the laser emits at both wavelengths single-mode and up to room temperature. We demonstrate its value for trace gas spectroscopy on simultaneous detection of $\mathrm{NO}$ and $\mathrm{NO}_{2}$, which, although spectrally separated by $300 \mathrm{~cm}^{-1}$, have been measured at sub-ppb levels.
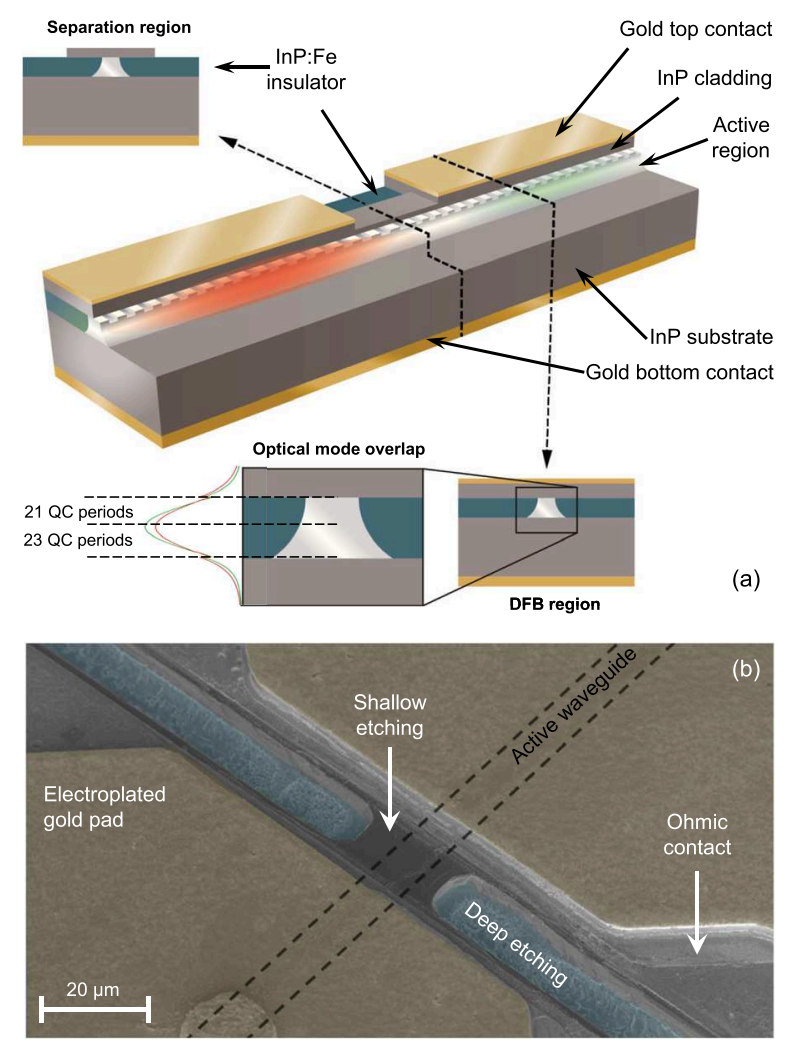

FIG. 1. (a) Schematic drawing of the dual-wavelength laser with crosssectional views of the injection and the separation regions. Calculated mode profiles of the fundamental TM modes supported by the active waveguides at $5.25 \mu \mathrm{m}$ (green) and $6.25 \mu \mathrm{m}$ (red) are also shown. (b) Top-view scanning electron microscope image of the separation region with the individual layers of the structure highlighted by false color. The dashed lines mark the position of the active waveguide. 
Figure 1(a) shows a schematic of the multi-wavelength laser processed in the buried heterostructure configuration. ${ }^{15}$ The QC material was grown on an InP substrate followed by a lithography step to define an $11 \mu \mathrm{m}$ wide and $5 \mathrm{~mm}$ long waveguide. The active waveguide is based on a heterogeneous cascade design, i.e., formed by a stack of two different types of bound-to-continuum single-wavelength active regions. The first consists of $21 \mathrm{In}_{0.635} \mathrm{Ga}_{0.365} \mathrm{As} / \mathrm{In}_{0.335} \mathrm{Al}_{0.665} \mathrm{As} \mathrm{QC}$ periods with layer sequence $44 / \mathbf{1 1} / 37 / \mathbf{1 1} / 36 / \mathbf{1 2} / 32^{2.1} / \underline{\mathbf{1 3}}^{2.6} / 29^{2.1}$ / $\mathbf{1 5}^{2.6} / 32 / 21 / 27 / 31 / 19 / 9 / 52 / 9 / 48 / 10$ optimized for emission at $\overline{6.25} \mu \mathrm{m}$, followed by $23 \mathrm{QC}$ periods designed for emission at $5.25 \mu \mathrm{m}$ with sequence $38 / \mathbf{1 2} / 33 / \mathbf{1 3} / 29 / \mathbf{1 4} / 26^{2.29} / \mathbf{1 5}^{2.74} / 24^{2.29}$ / $\mathbf{1 6}^{2.74} / 26 / \mathbf{2 3} / 23 / \mathbf{3 5} / 15 / \mathbf{1 0} / 45 / \mathbf{1 0} / 41 / \mathbf{1 1}$. The thicknesses (๕) of the InAlAs barriers are given in bold face and of the InGaAs wells in standard script, and the Si-doped layers are underlined with the doping concentrations $\left(10^{17} \mathrm{~cm}^{-3}\right)$ indicated in the superscript. The active region was designed using a homemade solver based on a density-matrix model, which predicts the second order gain and loss of the entire structure. ${ }^{6}$ It allowed us to minimize the cross-absorption between the individual gain regions, and, hence, to maintain good quantum efficiency and gain performance for both lasing wavelengths. Further, the combined active region was optimized to have similar current densities at both lasing thresholds to ensure good electronic transport through the whole structure.

A succession of two $2.5 \mathrm{~mm}$ long shallow-etched DFB gratings with different periodicities was defined on top of the active waveguide by deep-UV lithography to provide for single-mode emission at exactly $1600 \mathrm{~cm}^{-1}$ (front section) and $1900 \mathrm{~cm}^{-1}$ (back section). These two frequencies were chosen as they coincide with the strongest absorption bands of $\mathrm{NO}_{2}$ and $\mathrm{NO}$, respectively, which are relevant air pollutants and their simultaneous detection is of great practical interest. The ready waveguide was planarized with insulating Fe:InP and thereafter the top cladding was deposited in three layers; a low-doped Si:InP layer was first grown directly on top of the active region, followed by a highly doped Si:InP and a Si:InGaAs layer below the top metallic contact.

A special feature of our design is a process of electrical separation to isolate the neighboring laser sections. In the separation region, which is a $15 \mu \mathrm{m}$ long area between the front and the back sections, the entire highly doped top cladding and a large part of the low-doped Si:InP layer are etched away, leaving only a $3.5 \mu \mathrm{m}$ thin low-doped cladding layer on top of the active waveguide (see Figure 1(b)). The thin layer of high electric resistivity is kept to avoid reflections and scattering losses of the optical mode at the interface of the injection and the separation region. The resulting resistance between the laser sections is $65 \Omega$ at room temperature, which is much larger than the static resistance of the laser during operation (15 $\Omega$ ). Thus, current leakage between the two sections is sufficiently low to provide for absolute control over the emission wavelength; a single emission peak at $1600 \mathrm{~cm}^{-1}$ (resp. $1981 \mathrm{~cm}^{-1}$ ) is observed when injecting only into the back (resp. front) section, as depicted in Figure 2(a).

The dual-wavelength laser operates in pulsed mode in a wide temperature range up to $30^{\circ} \mathrm{C}$. As illustrated in Figure 2(b), the laser threshold current is similar for both emission wavelengths, being $0.8 \mathrm{~A}\left(3.6 \mathrm{kA} \mathrm{cm} \mathrm{cm}^{-2}\right)$ and $1.0 \mathrm{~A}$
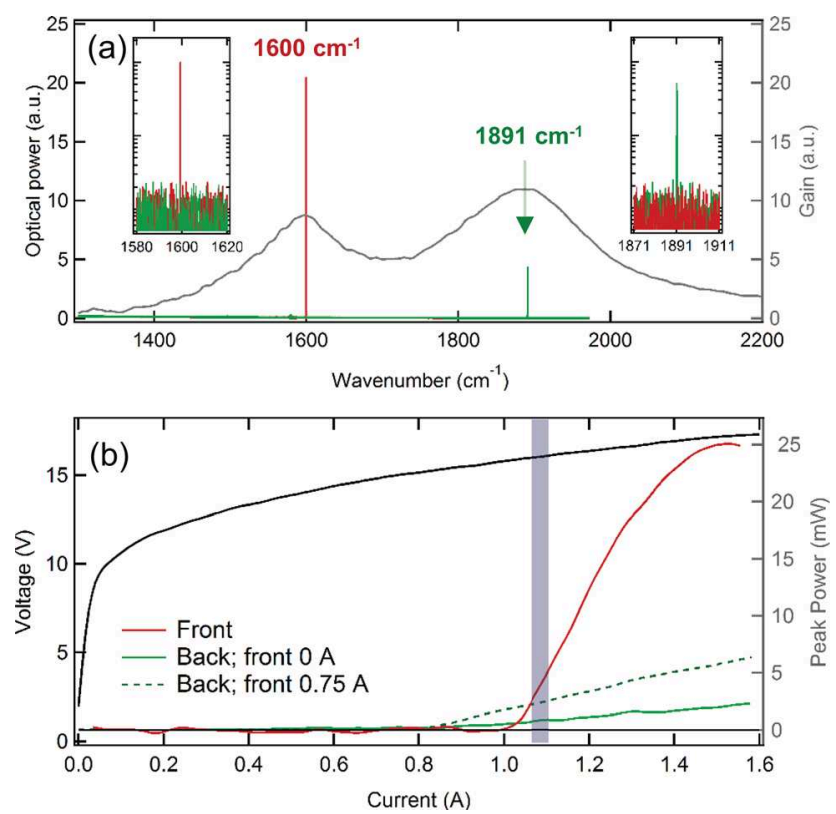

FIG. 2. (a) Laser emission spectrum defined by the front (red) and the back (green) DFBs, superimposed with the gain curve of the active QC material (gray) retrieved from the experimental electroluminescence spectra. Detail view of the emission peaks (insets) indicates no cross-talk between the respective laser sections. (b) Optical output power (red curve for the front and green for the back section) and voltage (black) as a function of the injected current. The optimal operating point of the laser for spectroscopy is also indicated (gray shaded region). When the front section is simultaneously biased with a sub-threshold current of $0.8 \mathrm{~A}$, a three-fold increase in the emitted power from the back laser section is achieved (green dashed).

$\left(4.4 \mathrm{kA} \mathrm{cm}^{-2}\right)$ at $1900 \mathrm{~cm}^{-1}$ and $1600 \mathrm{~cm}^{-1}$, respectively. The peak optical power, however, differs due to nonreciprocal laser geometry; while the $6.25 \mu \mathrm{m}$ emission from the front laser is directly coupled out of the active waveguide reaching $25 \mathrm{~mW}$ at $1.6 \mathrm{~A}$, the light from the back section first propagates through the front section where it gets attenuated in the unbiased active waveguide. This effect can be partially compensated by applying sub-threshold current to the front laser section, which reduces the propagation loss and increases the emitted power by a factor of 3 (see Figure 2(b)). The light extraction from the back laser section can be further increased by optimizing the physical lengths of the respective DFB sections, e.g., scaling their lengths with respect to the emission wavelength and mounting the laser such that the shorter section is positioned in the front. Yet, considering the sub- $\mu \mathrm{W}$ detection levels of the state-of-the-art MIR detectors, the $2.3 \mathrm{~mW}$ power achieved with an unbiased front laser section is largely sufficient for practical applications.

For the use in laser spectroscopy, the spectral position and the linewidth of laser emission have to be matched to the absorption lines of the targeted gas species. The challenge when using a dual-wavelength laser is to match the emission characteristics for both wavelengths simultaneously, and preferably under the same driving conditions. In our case, the laser emission can be tuned between $1595.5-1601.5 \mathrm{~cm}^{-1}$ and $1886.5-1894 \mathrm{~cm}^{-1}$, respectively, by changing the heatsink temperature from $30^{\circ} \mathrm{C}$ to $-20^{\circ} \mathrm{C}$. This tunable spectral range superimposed with the calculated absorption spectra of NO and $\mathrm{NO}_{2}{ }^{16}$ is shown in Figures 3(a) and 3(b). At a heatsink temperature slightly above $0^{\circ} \mathrm{C}$, the $5.25 \mu \mathrm{m}$ laser line 

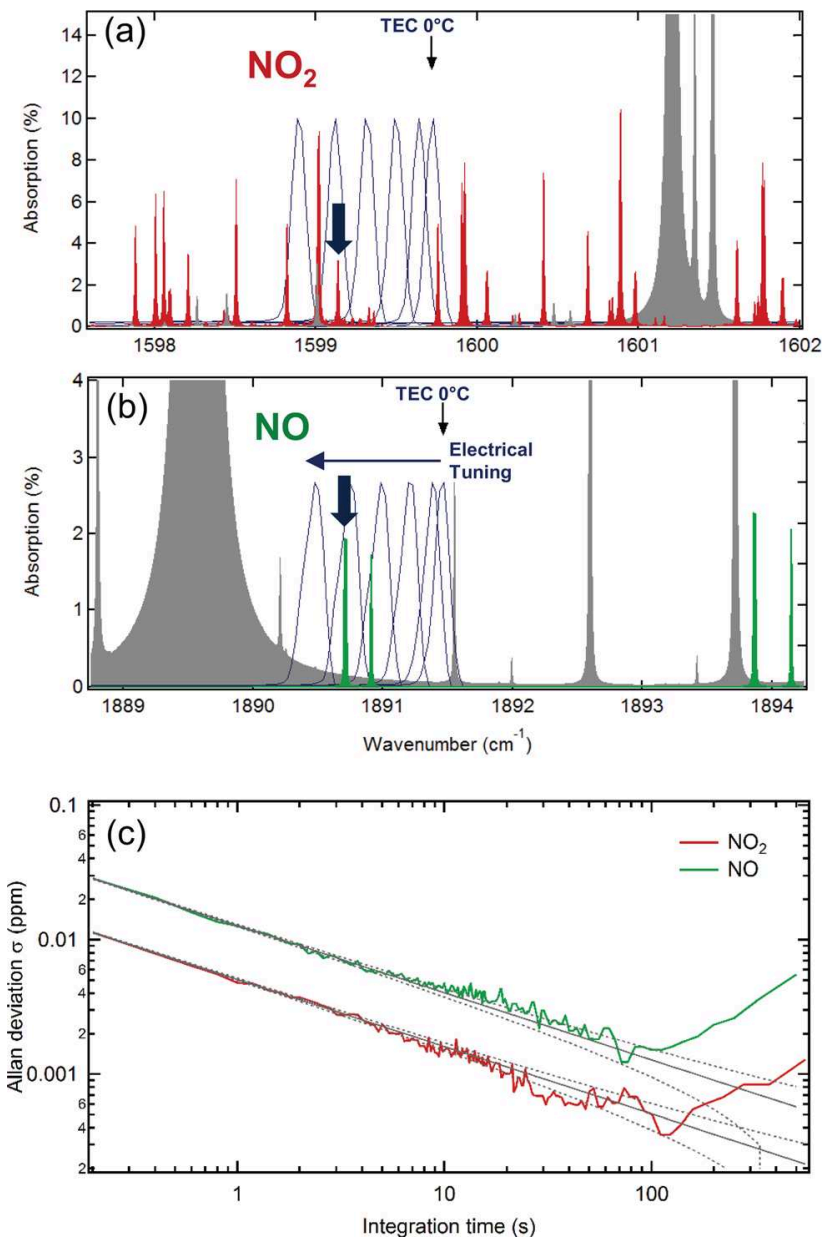

FIG. 3. Emission spectra of the front (a) and the back (b) laser section (blue) at a heatsink temperature of $0^{\circ} \mathrm{C}$ recorded by an FTIR spectrometer (Bruker Vertex 80 ) with $0.075 \mathrm{~cm}^{-1}$ resolution. The laser tuning range is overlaid with theoretical absorption spectra of $1 \mathrm{ppm} \mathrm{NO}_{2}$ (red), $1 \mathrm{ppm} \mathrm{NO}$ (green), and $1.3 \% \mathrm{H}_{2} \mathrm{O}$ (gray) simulated at $100 \mathrm{hPa}$ sample pressure and an optical pathlength of $36 \mathrm{~m}$; the absorption lines selected for the measurements are indicated by thick arrows. (c) Allan deviation plot associated to $\mathrm{NO}_{2}$ (red) and $\mathrm{NO}$ (green) concentration measurements.

overlaps with an intense absorption doublet of $\mathrm{NO}$ at $1890.71 \mathrm{~cm}^{-1}$ and the $6.25 \mu \mathrm{m}$ laser simultaneously probes a strong $\mathrm{NO}_{2}$ absorption line at $1599.14 \mathrm{~cm}^{-1}$, giving an optimal condition for the spectroscopy measurement.

It should be stressed that in respect to its spectral properties, the dual-wavelength laser matched the expectation in the very first process. This result, enabled by thorough numerical simulations of the whole structure, indicates that with the current know-how and the state-of-the-art fabrication technology it is feasible to produce matched multi-color lasers with a very high yield.

Once the heatsink temperature is set, fine tuning of the emission wavelengths across the two absorption lines (also shown in Figures 3(a) and 3(b)) is achieved electrically by applying a sub-threshold current ramp in addition to the laser driving pulses. This leads to further heating of the active region and to a rapid and well-controllable shift of the spectral emission.

As the multi-wavelength laser is operated in pulsed mode, the emission spectrum is inherently broadened due to a thermal chirp, which scales in magnitude with the driving current and the pulse duration. Spectrally narrow laser emission required for inter-pulse trace gas spectroscopy is thus achieved using very short pulses $(\leq 5 \mathrm{~ns})$ and by operating the laser at driving currents close to the lasing threshold. When driven with pulses of $5 \mathrm{~ns}$ at $1.1 \mathrm{~A}$, the laser linewidths determined from the measured gas spectra are $0.025 \mathrm{~cm}^{-1}$ at $6.25 \mu \mathrm{m}$ and $0.030 \mathrm{~cm}^{-1}$ at $5.25 \mu \mathrm{m}$, comparable to the fullwidth half-maximum of the targeted pressure-broadened absorption lines at $200 \mathrm{hPa}$.

The pulse-to-pulse amplitude variations measured at these driving conditions are $5 \%$ and $1 \%$, respectively. Being of similar magnitude as the traditional pulsed QC lasers, ${ }^{17}$ they mainly reflect the current fluctuations of the driving electronics.

The performance of the dual-wavelength laser was evaluated in a sensitive direct absorption spectroscopy setup with pulse intensity normalization, ${ }^{17}$ comparable to conventional pulsed systems for single-species detection. The setup is based on a 36-m astigmatic mirror Herriott multipass gas cell, ${ }^{18}$ where the investigated gas is sampled and the spectroscopic measurement takes place. The detection of the optical signal and the data acquisition was accomplished using a thermoelectrically cooled $\mathrm{HgCdTe}$ detector $(10 \mathrm{MHz}$ bandwidth, noise equivalent power of $1 \mathrm{pW} \mathrm{Hz}{ }^{-1 / 2}$ ) and a high-speed $50 \mathrm{MS} / \mathrm{s}$ digitizer card, synchronized with the laser pulser.

The dual-wavelength QC laser was operated at $1 \mathrm{MHz}$ pulse repetition rate, and swept in frequency with a subthreshold current ramp of $0.025-0.065 \mathrm{~A}$ at $10 \mathrm{kHz}$. Both the driving pulses and the ramp were generated using a single pulser unit, which output was time-division-multiplexed between the front and the back laser section with a digitally controllable electronic switch. The latter was operated at the rate of $200 \mathrm{~Hz}$, resulting in 47 spectra that were acquired and averaged after each switching event (the first three spectra were always discarded due to the down-time of the electronic switch). A custom-written LabView-based program provided for real-time fitting and quantification of the absorption spectra using molecular parameters from the HITRAN database. ${ }^{16}$

The sensitivity, linearity, and long term stability of the spectrometer were determined using gas cylinders with NO and $\mathrm{NO}_{2}$ at mixing ratios of $1 \mathrm{ppm}$. The gases were supplied into the multipass cell at $1 \mathrm{slpm}$ (standard liter per minute) and measured at a pressure of $80 \mathrm{hPa}$. The instrument performance was evaluated using Allan variance analysis, ${ }^{19}$ where the Allan deviation, which is a measure of the detection limit, is plotted as a function of the averaging time. The Allan plot (Figure 3(c)) associated with the $\mathrm{NO}_{2}$ and $\mathrm{NO}$ measurements indicates a $1 \sigma$-detection limit of 0.5 and $1.5 \mathrm{ppb}$ after $100 \mathrm{~s}$ of averaging. Although these values are slightly inferior to the performance of high-end pulsed QC lasers systems, ${ }^{20}$ the difference can be explained by a shorter optical pathlength and slower data acquisition rather than by the characteristics of the laser.

In conclusion, we presented a dual-wavelength DFB QC laser operating in time-multiplexed regime, which is suitable for a large number of spectroscopic applications ranging from environmental monitoring to industrial emission studies. The dual-wavelength laser combined with an electronic switch can operate at two wavelengths under the same driving conditions, which makes it compatible with any single-path laser spectroscopy setup. Hence, by simply replacing the laser source in readily available instrumentation, multiple species with 
spectrally distant absorption features can be detected. With this system, we demonstrate simultaneous measurement of $\mathrm{NO}$ and $\mathrm{NO}_{2}$ down to sub-ppb concentration levels, which is sufficient even for demanding applications in environmental and atmospheric research. Considering that only one output beam is emitted and no beam combining optical elements are needed, this development represents a significant step towards miniaturization of multi-species MIR sensors and has a strong potential to set a trend in MIR spectroscopy.

Future steps in the multi-wavelength laser development shall ultimately include the extension of the design to the full MIR spectral range, as well as combine three or more wavelengths. These further advances will be based on optimization of the active region design and the physical laser layout to obtain the highest possible quantum efficiency and minimal scattering loss. Additionally, more sophisticated driving schemes may be used, where the front sections are maintained below threshold and used as amplifiers or amplitude modulators. In short, we expect increasing performance and availability of multi-wavelength QC lasers, and thus rapid developments towards miniaturized multi-species laser spectroscopy.

This work is a part of the NanoTera-IrSens project supported by the Swiss National Research Foundation. The authors thank R. Brönnimann and A. Kunz for customizing the laser driving electronics for dual-wavelength operation.

${ }^{1}$ J. Faist, Quantum Cascade Lasers (Oxford University Press, Oxford, UK, 2013).
${ }^{2}$ J. Faist, F. Capasso, D. L. Sivco, C. Sirtori, A. L. Hutchinson, and A. Y. Cho, Science 264, 553 (1994).

${ }^{3}$ Y. Yao, A. J. Hoffman, and C. F. Gmachl, Nat. Photonics 6, 432 (2012).

${ }^{4}$ C. Gmachl, D. L. Sivco, R. Colombelli, F. Capasso, and A. Y. Cho, Nature 415, 883 (2002).

${ }^{5}$ C. Gmachl, D. L. Sivco, J. N. Baillargeon, A. L. Hutchinson, F. Capasso, and A. Y. Cho, Appl. Phys. Lett. 79, 572 (2001).

${ }^{6}$ R. Terazzi and J. Faist, New J. Phys. 12, 033045 (2010).

${ }^{7}$ A. Hugi, R. Maulini, and J. Faist, Semicond. Sci. Technol. 25, 083001 (2010).

${ }^{8}$ A. Hugi, G. Villares, S. Blaser, H. C. Liu, and J. Faist, Nature 492, 229 (2012).

${ }^{9}$ C. Gmachl, A. Straub, R. Colombelli, F. Capasso, D. L. Sivco, A. Sergent, and A. Y. Cho, IEEE J. Quantum Electron. 38, 569 (2002).

${ }^{10}$ B. G. Lee, M. A. Belkin, R. Audet, J. MacArthur, L. Diehl, C. Pflügl, F. Capasso, D. C. Oakley, D. Chapman, A. Napoleone, D. Bour, S. Corzine, G. Höfler, and J. Faist, Appl. Phys. Lett. 91, 231101 (2007).

${ }^{11}$ E. Mujagić, C. Schwarzer, W. Schrenk, J. Chen, C. Gmachl, and G. Strasser, Semicond. Sci. Technol. 26, 014019 (2011).

${ }^{12}$ M. Sidler, P. Rauter, R. Blanchard, P. Métivier, T. S. Mansuripur, C. Wang, Y. Huang, J.-H. Ryou, R. D. Dupuis, J. Faist, and F. Capasso, Appl. Phys. Lett. 104, 051102 (2014).

${ }^{13}$ L. Mahler, A. Tredicucci, F. Beltram, C. Walther, J. Faist, H. E. Beere, D. A. Ritchie, and D. S. Wiersma, Nat. Photonics 4, 165 (2010).

${ }^{14}$ A. Straub, C. Gmachl, D. Sivco, A. Sergent, F. Capasso, and A. Cho, Electron. Lett. 38, 565 (2002).

${ }^{15}$ M. Beck, D. Hofstetter, T. Aellen, J. Faist, U. Oesterle, M. Ilegems, E. Gini, and H. Melchior, Science 295, 301 (2002).

${ }^{16}$ L. S. Rothman, I. E. Gordon, Y. Babikov, A. Barbe, D. Chris Benner, P. F. Bernath, M. Birk, L. Bizzocchi, V. Boudon, L. R. Brown et al., J. Quant. Spectrosc. Radiat. Transfer 110, 533 (2009).

${ }^{17}$ D. Nelson, J. Shorter, J. McManus, and M. Zahniser, Appl. Phys. B 75, 343 (2002).

${ }^{18}$ J. B. McManus, P. L. Kebabian, and M. S. Zahniser, Appl. Opt. 34, 3336 (1995).

${ }^{19}$ P. Werle, R. Mücke, and F. Slemr, Appl. Phys. B 57, 131 (1993).

${ }^{20}$ J. B. McManus, M. S. Zahniser, D. D. Nelson, Jr., J. H. Shorter, S. Herndon, E. Wood, and R. Wehr, Opt. Eng. 49, 111124 (2010). 